\title{
Extracellular matrix proteins and displacement of cultured fibroblasts from duodenal biopsies in celiac patients and controls
}

\author{
Leda Roncoroni ${ }^{1,3 *}$, Luca Elli ${ }^{1}$, Maria Teresa Bardella ${ }^{1}$, Gianluca Perrucci ${ }^{2}$, Michele Ciulla ${ }^{2}$, Vincenza Lombardo ${ }^{1}$, \\ Carolina Tomba ${ }^{1}$, Dario Conte ${ }^{1}$ and Luisa Doneda ${ }^{3}$
}

\begin{abstract}
Background: Celiac disease (CD) is mainly characterised by villous atrophy and mucosal architectural rearrangement. The fibroblasts (FBs) are the most abundant mesenchymal cell type in the intestinal mucosa and are responsible for both the architectural arrangement of the villi and the formation of the extracellular matrix (ECM). This study aimed at the evaluation of both the intracellular distribution of different proteins involved in ECM and FBs characterisation, and the cellular displacement of primary FBs obtained from duodenal endoscopic biopsies of healthy subjects and celiac patients.

Methods: Primary healthy and celiac duodenal FBs were evaluated by means of immuno-fluorescence assay for collagen type I and IV, fibronectin, actin, alpha-Smooth Muscle Actin (alpha-SMA), Fibroblast Surface Protein (FSP) and transglutaminase type 2 (TG2). The geometric indexes of the fluorescence signals were investigated by image analysis software (Image J, NIH). Both morphology and kinetic were evaluated during a 72 hours time course movie. TG2 medium activity was evaluated by means of ELISA.

Results: All the cells examined were immunopositive for FSP, alpha-SMA, actin, collagen I, collagen IV and TG2. CD cells showed a signet collagen-I and collagen-IV pattern, as compared to the controls being characterised by a spindle geometry. Moreover, the collagen signals in CD FBs showed a significantly higher circularity index (major orthogonal diameter ratio) than the controls ( $p<0.0001)$, whereas the perimeter and area ratio were significantly lower $(p<0.0001)$. The TG2 signal had a decreased area $(p<0.05)$, but a two-fold increased medium activity. The time course highlighted a reduction of the displacement of CD FBs.

Conclusions: The isolated primary CD FBs showed a different collagen and TG2 pattern of distribution associated with a different cellular displacement. The reasons for such CD cell peculiar characteristics are yet unknown but they might represent a factor in the progression of the intestinal damage.
\end{abstract}

Keywords: Celiac disease, Fibroblasts, Extracellular matrix, Collagen, Tissue transglutaminase, Transglutaminase type 2

\section{Background}

The small bowel mucosal architecture is maintained through a delicate balance among cell production in the crypt compartment, enterocytes migration along the villi, and extrusion of mature epithelial cells from the tip of the villi into the intestinal lumen [1]. This continuous

\footnotetext{
* Correspondence: leda.roncoroni@tiscali.it

${ }^{1}$ Center for the Prevention and Diagnosis of Celiac Disease, Gastroenterology Unit II, Fondazione IRCCS Ca' Granda - Ospedale Maggiore Policlinico and Università degli Studi di Milano, Via F. Sforza 35, Milan 20122, Italy

${ }^{3}$ Department of Biomedical, Surgical and Odontoiatric Sciences, Università degli Studi di Milano, Via Festa del Perdono 7, Milan 20122, Italy

Full list of author information is available at the end of the article
}

process of cellular differentiation and natural apoptosis arises and is controlled by interactions among epithelium, mesenchymal cells, gut associated lymphoid tissue and the structure of the extracellular matrix (ECM), through mechanisms still largely unknown [2]. Among the different cell types resident in the intestinal mucosa, the fibroblasts (FBs) are the most represented mesenchymal ones and are responsible for both the architectural arrangement of the villi and the formation of the ECM, mainly composed by collagen fibrils [3,4]. As concerns the intestinal wall, ECM (i.e. the structural basis of the human tissues) includes the basement membrane that sustains the epithelium and the

\section{(O) Biomed Central}


villous axis [5]. ECM is mainly formed by fibrillar collagen and provides the support for the different cell types resident in the mucosa (type I collagen) and for the epithelial line of enterocytes forming the intestinal barrier (type IV collagen). ECM also includes the glycoproteins (fibronectin), connecting collagen and cells. All the proteins participating in ECM are interconnected to form a three-dimensional structure by enzymes. The most important of such enzymes is the transglutaminases type 2 (TG2), capable to catalyze stable isopeptide bonds in physiological conditions characterized by neutral $\mathrm{pH}$ and low calcium concentrations [6]. Collagen and enzymes are secreted by FBs, which also participate in the tissue re-modelling and repair through their movements along the protein fibrils of the ECM, secreting molecules when and where needed and in association with the other resident cells. Besides this tissutal morphogenetic function FBs actively participate in the inflammatory response by producing chemokines and cytokines, and in the antigen presentation by acting as non-professional antigen presenting cells [7-12]. Moreover, in the autoimmune processes, FBs are frequently targeted by autoantibodies, leading to a biological dysfunction $[9,13,14]$. Based on the above characteristics, FBs are involved in diseases characterized by tissue architectural alterations, inflammation and autoimmunity.

In the celiac disease (CD), which is the most common autoimmune enteropathy in Western countries, gluten ingestion progressively leads to villous atrophy, crypt hyperplasia with a two to three-fold increased depth of the lamina propria, an increased number of immunological cells (B and T lymphocytes, macrophages and eosinophils) and intraepithelial lymphocytosis $[6,15]$. These abnormalities, responsible for any functional derangement, are ascribed to the primary cytotoxic effects of gliadin and IgA autoantibodies against TG2 on the surface of FBs and in the ECM [16-18]. In particular, Verbeke et al. reported a derangement of collagen distribution in the duodenal mucosa of CD subjects [16]. As of earlier studies by the Authors [19], duodenal CD FBs can be successfully cultured; they are morphologically different from healthy FBs and potentially carry intrinsic alterations, suggesting their primary involvement in both the $\mathrm{CD}$ pathogenesis and the development of the atrophic duodenal lesions.

The present study was aimed at evaluating, in consecutive naif $\mathrm{CD}$ patients and matched controls, the cellular distribution of type I and IV collagens, fibronectin, actin, TG2 and the cellular displacement of primary $\mathrm{FBs}$ obtained from duodenal endoscopic biopsies.

\section{Methods \\ Patients}

From February 2009 to December 2011, 50 consecutive subjects selected from a cohort of patients undergoing upper GI tract endoscopy for suspected CD, who gave their written informed consent to the study, were enrolled: there were $22 \mathrm{CD}$ patients (10 males and $12 \mathrm{fe}-$ males, mean age 45 , range $35-55)$ and 28 non-CD controls (CTRs) (16 males and 12 females, mean age 42, range 30-60). The $C D$ diagnosis was based on the serological presence of anti-TG2 IgA (ELISA or radio-immunoassay tests) and/or anti-endomysium IgA (immunofluorescence technique) auto-antibodies and, at least, a Marsh-Oberhuber grade III duodenal histology. The nonCD group was composed of serologically negative subjects without endoscopic or histological lesions, not reporting other autoimmune or intestinal diseases.

The study plan was approved by the Ethics Committee of the "Fondazione IRCCS Cà Granda-Ospedale Maggiore Policlinico".

\section{Duodenal specimens and cell cultures}

Three duodenal biopsies obtained from all the participants by standard endoscopic forceps (Boston Scientific, USA) were rapidly dipped into sterile tubes (Becton and Dickinson, Italy) containing an appropriate medium.

The samples were finely chopped with disposable surgery knives and incubated in Ham's F12 medium (Euroclone, Italy), containing liberase blendzyme 2 $(1.4 \mathrm{~W} / \mathrm{mL})$ (Roche, Italy) and the cells so derived cultured. Viability was routinely checked by a trypan blue dye exclusion assay (Sigma, Italy) and only those cultures with viability $>95 \%$ were used. Mycoplasma contamination was routinely checked and ruled out using the Hoechst method.

\section{Immunocytochemistry}

The different types of proteins were analysed by immunocytochemistry: characterising proteins i.e., the "FB surface protein" (FSP, monoclonal anti-human FSP, Clone 1B10; Sigma, Italy), the $\alpha$-smooth muscle actin ( $\alpha$ SMA) (ABCAM, Italy) and actin (Sigma, Italy), and the ECM components and enzymes i.e., the type I and IV collagens (ABCAM, Italy), fibronectin (Sigma, Italy) and TG2 (Zedira, Germany). Primary antibodies were used following the manufacturer's recommended dilutions. Cells, seeded onto 24-well plates at a concentration of 20,000 cells/plate, were washed twice 48 hours later in PBS and fixed with $3.7 \%$ formaldehyde in PBS for 15 minutes at room temperature (RT). Fixed cells were permeabilised with $0.1 \%$ triton X-100 (Sigma, Italy) in PBS for 15 minutes at RT. The non specific binding of any secondary antibody was blocked by incubation with normal foetal serum for 30 minutes at RT. The immunostaining cells were then rinsed with PBS and incubated with a fluorochrome conjugated secondary antibody for 45 minutes at RT, according to the donor species of the primary antibodies. PBS was used as the 
negative control in place of the primary antibody. Counter-staining was performed using DAPI; the glass coverslip was mounted on glass slides with ProLong Gold Antifade Reagent (Invitrogen, Italy). Images were obtained by fluorescence microscope (Leica, Italy).

The epithelial cellular type was carefully excluded in performing cytokeratine analysis by using anti-human Cytokeratine 20 (Sigma, Italy).

\section{Medium transglutaminase activity}

TG activity was evaluated by a colorimetric technique (Covalab, France) according to the manufacturer's instructions. Briefly, $50 \mu \mathrm{L}$ of the cell culture medium was dispensed in each well of the 96-well microtiter plate with covalently coupled CBZ-Gln-Gly and incubated with calcium, dithiothereitol and biotinylated cadaverine; strepavidin-labelled peroxidase was added to the wells and the peroxidase activity revealed using $\mathrm{H}_{2} \mathrm{O}_{2}$ and tetramethyl benzidine. Optical density was measured at $450 \mathrm{~nm}$ with a microplate reader (Bouty Diagnostici, Italy). The results were normalized referring to the cell number.

\section{Image analysis}

The digital images were analysed as previously described $[19,20]$. Briefly, after the localization of fluorescence signals, multiple adjacent high-power fields in each section were selected, acquired, and stored in a personal computer (Power Mac G4, 867 MHz, 640 MB RAM, Apple, USA) in JPEG format (5:1) at 32 bits/pixel on a $764 \times 560$ pixel matrix. The stored images were then read by an expert blinded to both image sequence and assignment, and analyzed to determine the differences between $C D$ and CTRs FBs. A 3D interactive surface plot was digitally reconstructed for every image and based on a 256level scale (0-255).

Fluorescent signal analysis was performed on the digitally elaborated images. Analysis algorithms were developed as a set of macros executed with NIH Image (Interactive 3D Surface Plot Plugin), which is an integrated image-processing software distributed as freeware by the National Institutes of Health (Bethesda, USA). Prior to analysis an automated threshold process was performed on the images to minimize the influence of light variation in the microscope field and in the operator's subjective settings. This process cuts off any object below the minimum signal intensity. FBs were recognized on the basis of their size and intensity signal using a cell count algorithm which draws a region of interest (ROI) around each discrete object within the image. The minimum size in pixels of the objects to be included in the count was previously defined by accurately measuring twelve representative FBs. Objects below the minimum size were not included in the analysis. The evaluation of the fluorescent signal included, on one hand, the major orthogonal diameters and their ratio as index of circularity and, on the other hand, the perimeter, the area and their ratio, as index of complexity.

\section{Shape and motility evaluation}

Morphology and growth were evaluated during a 72 hour time course movie. Images were acquired with a Leica DMI AF6000LX microscope at 10× magnification.

After three days from seeding, the cell culture plates (7 CD and 7 CTRs) were placed under the microscope connected to a camera. The camera was set for capturing frames every 10 minutes over 72 hours. The compressed frames were composed into the experimental movies (one for each plate); the movies were accelerated by software (LAS-AF imaging software), obtaining a 40 seconds long one. Thus, one second of the final digital movie corresponded to a 1.8 hours long period of culture. Fifteen randomly selected cells from 3 different movies from each group were selected at time 0 (i.e. at the beginning of the movie). The nucleus was fixed to track the displacement after 12, 24, 36, 48, 60 and 72 hours (Photoshop Adobe, USA). Data were expressed as total displacement $(\mu \mathrm{m})$ and velocity $(\mu \mathrm{m} / \mathrm{h})$. Also perimeter, circularity index and feret diameters were analysed as previously shown at time 0 and after 36 and 72 hours.

\section{Statistical analysis}

Data were expressed as mean \pm standard deviation (SD) or median and range. Comparisons of the parameters were carried out by one-way ANOVA. Statistical analysis was performed using statistical software (SPSS version 13 , SPSS, USA), considering a $\mathrm{p}$ value $<0.05$ as significant.

\section{Results}

Both CD and CTR cells expressed FSP, $\alpha$-SMA, actin, type I and IV collagens, fibronectin and TG2 (Figure 1) but not cytokeratine 20. CD cells showed a signet type I and IV collagen patterns, whereas CTRs had a spindle geometry (Figure 1). Type I and IV collagen morphometric analysis, performed on CD and CTR FBs obtained on the same day of culture, showed some differences. As detailed in Table 1 and Figure 2, which shows the digitally elaborated images, the collagen fluorescent signal involved a larger part of the FB cytoplasm with an increased circularity index in CD FBs compared to CTR ones. Statistically significant differences between the TG2 fluorescent signal of CD and CTR FBs were found. In particular, in the CD FBs the area covered by the TG2 fluorescent signal decreased by $50 \%$ (Table 2), while TG activity increased in the culture medium of $C D$ FBs (Figure 3). No statistically different findings were 


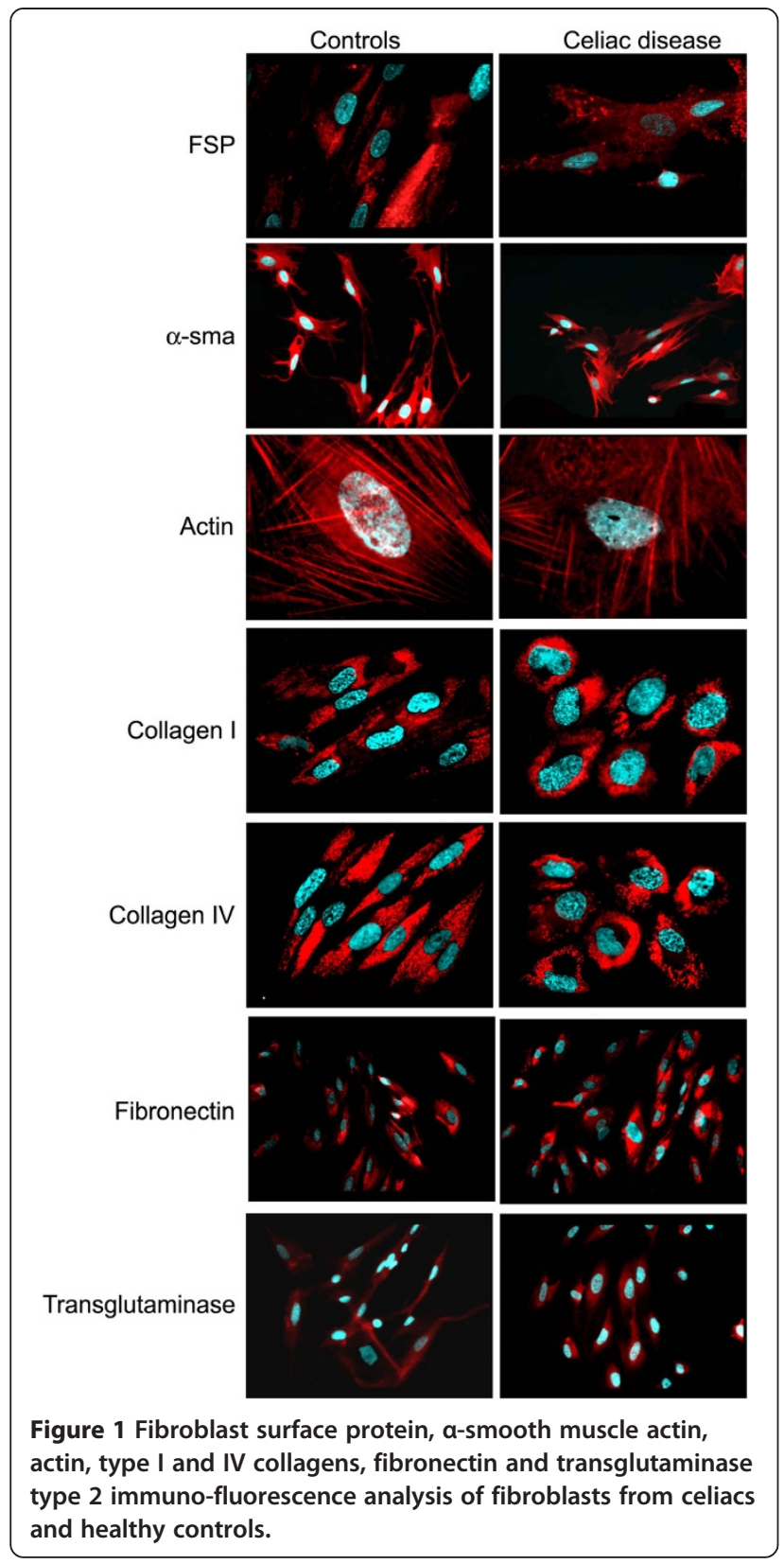

Table 1 Morphometrical characteristics of intracellular fluorescent signal of type I and IV collagen fibroblasts from celiacs and healthy controls

\begin{tabular}{|c|c|c|c|c|c|c|}
\hline \multirow[b]{2}{*}{ Parameter } & \multicolumn{3}{|c|}{ Type I collagen } & \multicolumn{3}{|c|}{ Type IV collagen } \\
\hline & Controls (\#12) & Celiacs (\#12) & $P$ value & Controls (\#12) & Celiacs (\#12) & $P$ value \\
\hline Feret Diameter $(\mu \mathrm{m}) *$ & $53.00 \pm 11.00$ & $35.20 \pm 5.00$ & $<0.005$ & $62.90 \pm 12.05$ & $32.90 \pm 4.90$ & $<0.0001$ \\
\hline Perimeter $(\mu \mathrm{m})$ & $121.30 \pm 24.00$ & $107.60 \pm 16.80$ & $<0.005$ & $145.90 \pm 27.20$ & $99.60 \pm 12.06$ & $<0.001$ \\
\hline Area $\left(\mu m^{2}\right)$ & $377.20 \pm 86.90$ & $563.40 \pm 122.80$ & $<0.05$ & $375.60 \pm 118.60$ & $502.70 \pm 98.10$ & $<0.05$ \\
\hline Circularity index ${ }^{\circ}$ & $0.30 \pm 0.10$ & $0.62 \pm 0.11$ & $<0.005$ & $0.20 \pm 0.06$ & $0.60 \pm 0.11$ & $<0.0001$ \\
\hline Complexity index $(1 / \mu m)^{\&}$ & $0.30 \pm 0.03$ & $0.19 \pm 0.02$ & $<0.0001$ & $0.40 \pm 0.08$ & $0.20 \pm 0.03$ & $<0.0001$ \\
\hline
\end{tabular}

* Feret diameter: longest axis length.

- Circularity index: Feret diameter/short axis length.

\& Complexity index: perimeter/area $(1 / \mu \mathrm{m})$. observed for $\alpha$ SMA, actin and fibronectin signals (data not shown). The cellular shapes of CD and CTR FBs are detailed in Table 3.

The CD FBs showed a decreased motility and velocity. In detail, during the 72 hours period of the observed culture the CD FBs moved $113 \pm 39 \mu \mathrm{m}$ while the CTR FBs $222 \pm 88 \mu \mathrm{m}$ with a velocity of $1.5 \pm 0.5 \mu \mathrm{m} / \mathrm{h} v s$ $3.0 \pm 1.2 \mu \mathrm{m} / \mathrm{h}$, the difference being statistically different (see Figure 4 for movement tracking). Two additional movie files show the FBs displacement in more detail S2 for CD and CTR FBs respectively).

\section{Discussion} an alteration of the collagen and TG2 immunofluorescence signal in the CD FBs compared to the CTR ones. These abnormalities could be involved in the decreased motility observed during the time-course experiments and in turn in the villous damage observed in CD.

The maintenance of a normal duodenal mucosal morphology results from a continuous interaction between the epithelium, the ECM represented by the basement membrane and the underlying FBs network, responsible for the secretion of collagen molecules and matrix stabilizing enzymes (TG2) [6]. In CD the mucosal structure is deeply altered as proven by the typical "stigmata" of CD represented by mucosal atrophy, crypt hyperplasia and lymphocytic infiltration. Also the lamina propria, composed by type IV collagen and controlling the interface between the epithelium and the subepithelial compartment, is altered in CD $[21,22]$. These aforementioned ECM alterations can be attributed to the secreted autoantibodies (anti-TG2 and anti-gliadin) interacting with the matrix proteins or alternatively to a primary defect of CD FBs which could abnormally express these proteins or enzymes [23]. Moreover, FBs control the degradation of ECM through the secretion of lytic enzymes, as the metalloproteases (MMP), and their mechanisms can play a role in the CD progress. Schuppan et al. for example have demonstrated that ECM could act (Additional file 1: Video S1 and Additional file 2: Video

The results from the present study are consistent with inhibitors [24-26]. Previous findings suggest that both these 


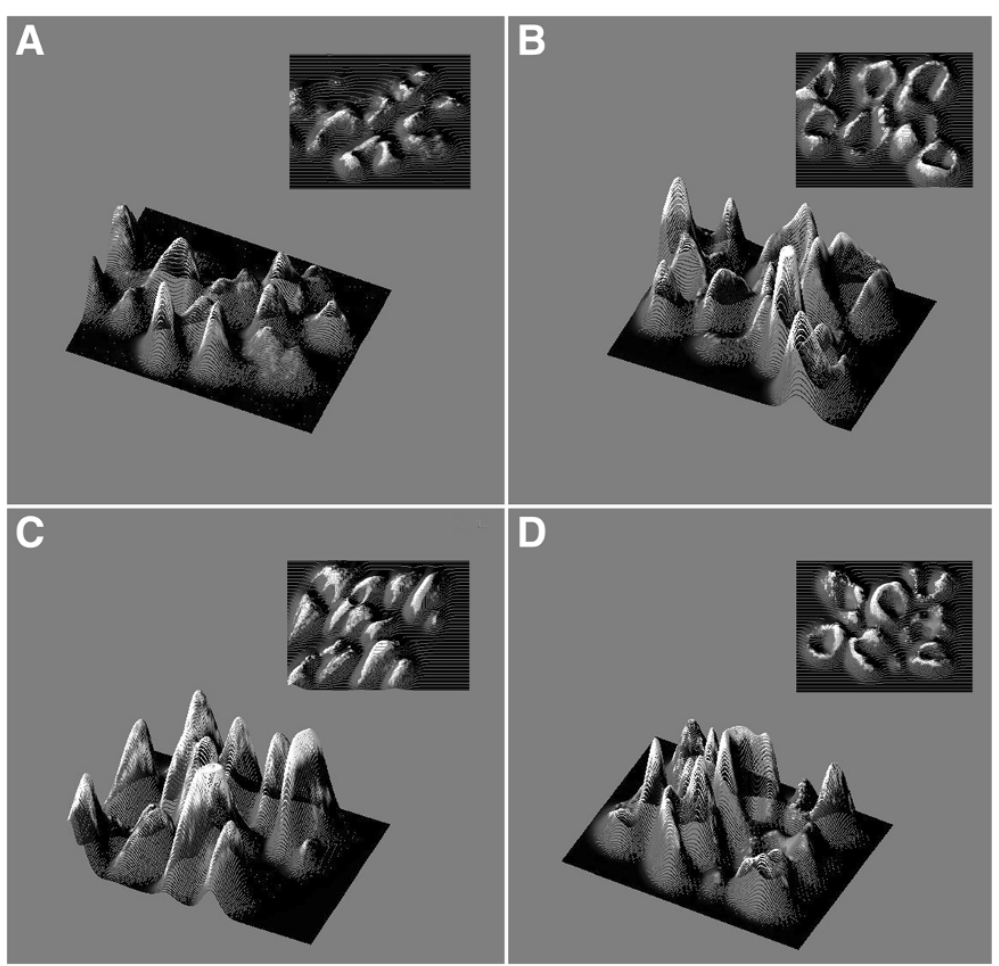

Figure 2 Digitally reconstructed images from immunofluorescence analysis of type I and type IV collagen of fibroblasts from healthy controls (panels A and C) and celiacs (panels B and D), respectively.

as a reservoir of autoantibodies, fuelling the mucosal inflammation and eventually modifying the $\mathrm{pH}$ and calcium concentrations, making the environment suitable for protein lysis and TG2 activation in terms of switching from isopeptide bonds to the deamidation using $\mathrm{H}_{2} \mathrm{O}$ as acyl acceptor $[6,23]$. On the other hand, Verbeke et al. have reported a decreased immunofluorescence signal of type IV collagen and laminin with a "leaky" basement membrane [16]. Accordingly, the increase of type I and IV collagen signals in FBs and the increase of TG2 activity in the medium could represent an attempt of CD FBs to restore the decreased collagen levels in the ECM of CD patients and to increase the formation of isopeptide bonds stabilizing the matrix fibrils. Although demonstrated only in vitro this

\section{Table 2 Morphometrical characteristics of the} intracellular fluorescent signal of the transglutaminase type $\mathbf{2}$ in fibroblasts from celiacs and healthy controls

\begin{tabular}{lrrr}
\hline Parameter & Controls (\#12) & Celiacs (\#12) & P value \\
\hline Feret Diameter $(\mu \mathrm{m})^{*}$ & $26.60 \pm 5.40$ & $19.10 \pm 4.40$ & $<0.05$ \\
Perimeter $(\mu \mathrm{m})$ & $73.00 \pm 23.10$ & $53.10 \pm 13.80$ & $\mathrm{NS}$ \\
Area $\left(\mu \mathrm{m}^{2}\right)$ & $143.90 \pm 50.70$ & $90.00 \pm 34.80$ & $<0.05$ \\
Circularity index $^{\circ}$ & $0.38 \pm 0.20$ & $0.40 \pm 0.10$ & $\mathrm{NS}$ \\
${\text { Complexity index }(1 / \mu \mathrm{m})^{\&}}^{*}$ & $0.50 \pm 0.10$ & $0.60 \pm 0.20$ & $\mathrm{NS}$ \\
\hline
\end{tabular}

* Feret diameter: longest axis length.

- Circularity index: Feret diameter/short axis length.

\& Complexity index: perimeter/area $(1 / \mu \mathrm{m})$; NS, Not significant. scenario could also be present in the flattened CD mucosa; in other words, as primary cell cultures maintain the memory of their site of origin even after different passages, a restoration of the original in vivo scenario could be possible $[27,28]$. To underline these mechanisms, the fibronectin levels did not differ between $\mathrm{CD}$ and healthy FBs, in line with the results obtained by Korhonen et al. who reported a comparable pattern both in $\mathrm{CD}$ and non-CD duodenal mucosa [29].

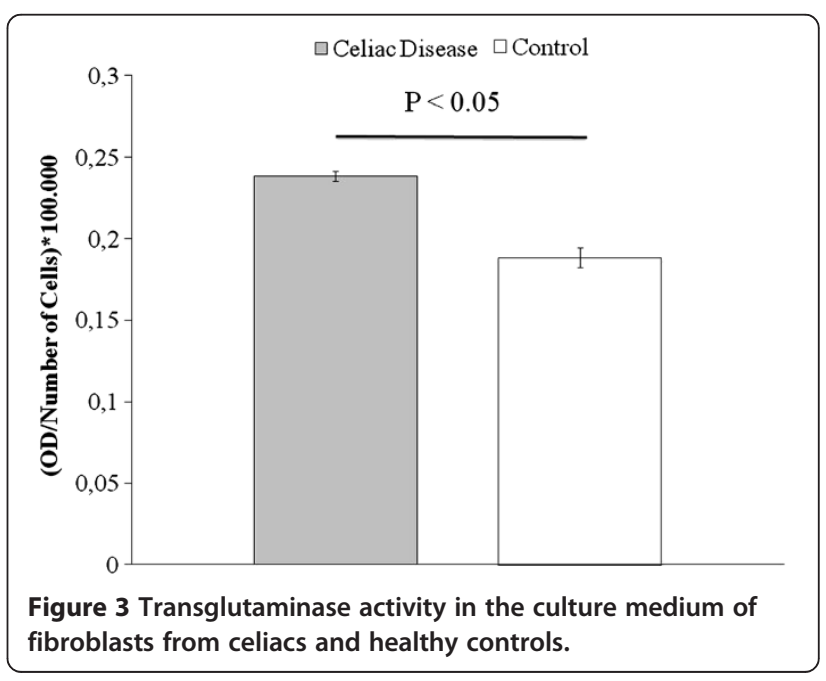


Table 3 Morphometrical characteristics of fibroblasts from celiacs and healthy controls

\begin{tabular}{lrrr}
\hline & \multicolumn{3}{c}{ Characteristics of fibroblasts } \\
\cline { 2 - 4 } Parameter & Controls (\#12) & Celiacs (\#12) & P value \\
\hline Feret Diameter $(\mu \mathrm{m})^{*}$ & $62.90 \pm 21.60$ & $89.00 \pm 28.00$ & $<0.05$ \\
Perimeter $(\mu \mathrm{m})$ & $188.30 \pm 61.90$ & $252.30 \pm 99.80$ & $<0.05$ \\
Area $\left(\mu \mathrm{m}^{2}\right)$ & $744.60 \pm 357.60$ & $933.60 \pm 649.30$ & NS \\
Circularity index $^{\circ}$ & $0.30 \pm 0.20$ & $0.20 \pm 0.10$ & NS \\
${\text { Complexity index }(1 / \mu \mathrm{m})^{\&}}^{*}$ & $0.30 \pm 0.10$ & $0.40 \pm 0.20$ & NS \\
\hline
\end{tabular}

* Feret diameter: longest axis length.

- Circularity index: Feret diameter/short axis length.

\& Complexity index: perimeter/area $(1 / \mu \mathrm{m}) ; N S$, Not significant.

In the present study FBs resulted positive for $\alpha$ SMA, which is the most relevant marker of myofibroblasts (mFBs) and represents an intermediate state between FBs and smooth muscle cells [30]. As observed in our experiments, $\mathrm{mFBs}$ display prominent cytoplasmatic actin microfilaments (stress fibers) and are connected to one another by adherent and gap junctions [10]. In the gastrointestinal tract two kind of mFBs are usually recognized, the $\alpha$-SMA positive and those negative, resident in the apical and basal parts of the villi, respectively [30]. In detail, $\alpha$-SMA positive FBs correspond to our in vitro population and are involved in both epithelial differentiation and ECM formation [12]. More recently, using immunohistochemistry, some authors have demonstrated that $\alpha$-SMA positive subepithelial mFBs constitutively express class II MHC molecules and are distinct from professional antigen presenting cells [31].

A further interesting finding from the present series, reported for the first time, is represented by the decreased motility of CD FBs compared with the control ones, as indicated by a $50 \%$ reduction of the displacement of CD FBs during the 72-hour movie recording. This finding refers to different biological aspects, as cell shape/dimension and migration are dynamic processes connected to the cellular fate (growth/death) and depending on the ECM composition and endocellular cytoskeleton. Thus, round shapen cells (circularity index $=1$ ) appear as in stationary state while a Y-like shape as frequently observed in FBs cultures (circularity index $<1$ ) characterizes those cells ready for migration [32]. In fact, the "Y" shape is the most favorable for polarized migration, with a frontal adhesion point and a rear arc where active forces as given by the Laplace law on tension, prepare for the movement of the cell [32]. The molecular mechanisms underlying cellular motility are largely unknown, even if the Rho family of GTPases seems to play a crucial role [33]. Beyond the complex molecular processes connected to FB motility, several relevant clinical consequences have been reported. In particular, decreased FB motility has been reported in patients affected by diabetes mellitus (DM): for such patients a

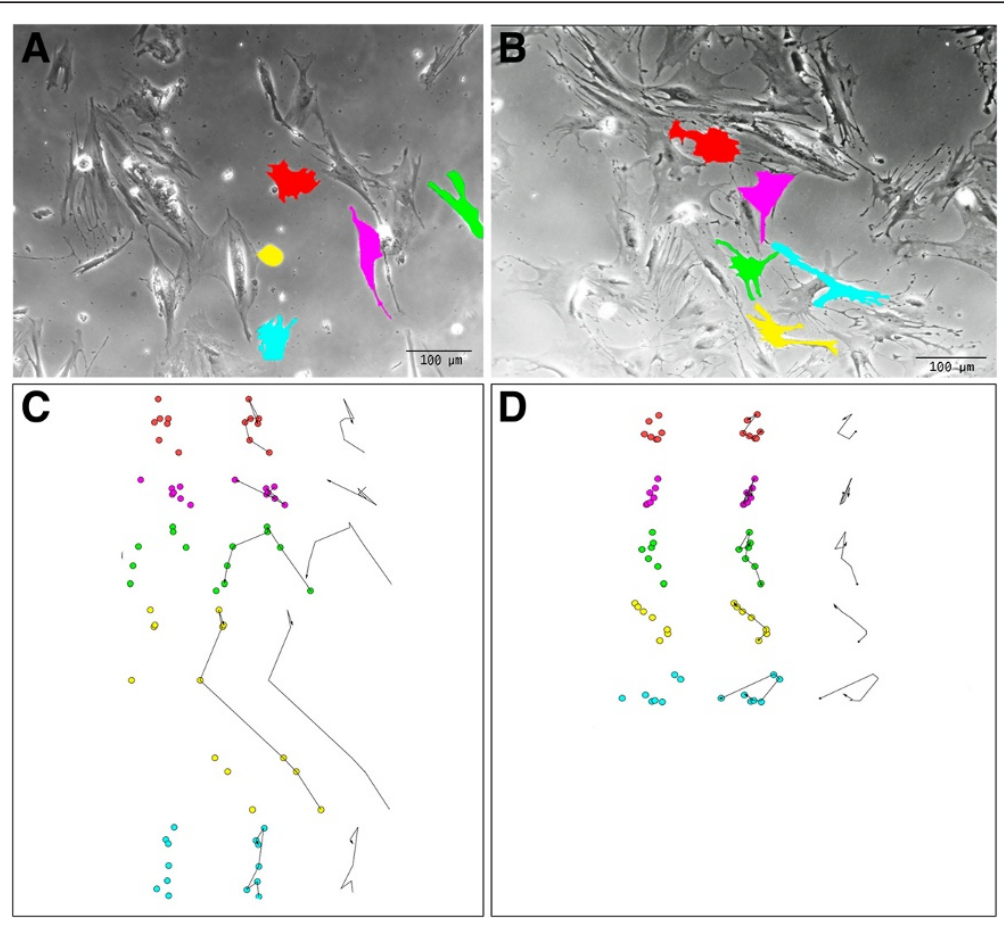

Figure 4 Motility tracking of fibroblasts from healthy controls (panel A) and celiacs (panel B). In the upper panels the images at the beginning of the movie recording are reported with the cells tracked. In the lower panels the movement of the cell nuclei into the field after 12 , $24,36,48,60$ and 72 hours are reported with the final trace. 
migratory defect represents a relevant impairment for wound healing, responsible for chronic skin ulcerations even requiring amputation [34]. The discussed finding could represent a connection between $C D$ and $D M$, which are supposed to present some common factors such as genetic background. Moreover, DM animal models are currently used to investigate particular aspects of $\mathrm{CD}$ as they are capable to develop duodenal atrophy when exposed to gluten feeding [35]. It remains an interesting point for future investigation whether the observed alterations are common to other gastrointestinal autoimmune disorders (inflammatory bowel disease, autoimmune enteropathy) or they could be reverted in case of FBs derived from $\mathrm{CD}$ non atrophic mucosa, where a different FB population has been selected from duodenal micro-environment.

\section{Conclusion}

Overall, the results from the present study highlight the importance of FBs in $\mathrm{CD}$ pathogenesis, strongly supporting the need for further investigations on this topic in order to improve our understanding of the $\mathrm{CD}$ related injury processes. Transitionally, if the defect of tissue repair process in CD FBs should be confirmed as pivotal in the normalization of duodenal mucosa after gluten withdrawal from a patient's diet, the possibility of ECM targeted therapies could play a role in the $\mathrm{CD}$ research.

\section{Additional files}

Additional file 1: Video S1. CD FBs.

Additional file 2: Video S2. CTR FBS.

\section{Competing interests}

The authors declare that they have no competing interests.

\section{Authors' contributions}

$L R$ and $V L$ perfomed the cell cultures, immunofluorescence eperiments and ELISA. GP and MC dealt with the FB movies and image analysis. LE and CT planned the study, dealt with the patients and performed upper endoscopies with duodenal biopsies. MTB, DC and LD analyzed data and prepared the manuscript. All the authors read and approved the final manuscript.

\section{Acknowledgements}

We are deeply indebted to Professor Elena Cattaneo of the Department of BioSciences at Università degli Studi di Milano - Milan, Italy for granting access to use the DMI6000LX microscope.

\section{Author details}

${ }^{1}$ Center for the Prevention and Diagnosis of Celiac Disease, Gastroenterology Unit II, Fondazione IRCCS Ca' Granda - Ospedale Maggiore Policlinico and Università degli Studi di Milano, Via F. Sforza 35, Milan 20122, Italy. ${ }^{2}$ Department of Clinical and Community Sciences, Università degli Studi di Milano, Via F. Sforza 35, Milan 20122, Italy. ${ }^{3}$ Department of Biomedical, Surgical and Odontoiatric Sciences, Università degli Studi di Milano, Via Festa del Perdono 7, Milan 20122, Italy.
Received: 13 January 2013 Accepted: 23 March 2013

Published: 8 April 2013

\section{References}

1. MacDonald TT, Monteleone I, Fantini MC, Monteleone G: Regulation of homeostasis and inflammation in the intestine. Gastroenterology 2011, 140:1768-1775

2. Burke JP, Mulsow JJ, O'Keane C, Docherty NG, Watson RW, O'Connell PR: Fibrogenesis in Crohn's disease. Am J Gastroenterol 2007, 102:439-448.

3. Powell DW, Pinchuk IV, Saada Jl, Chen X, Mifflin RC: Mesenchymal cells of the intestinal lamina propria. Annu Rev Physiol 2011, 73:213-237.

4. Simon-Assmann P, Kedinger M, De Arcangelis A, Rousseau V, Simo P: Extracellular matrix components in intestinal development. Experientia 1995, 51:883-900.

5. Shi YB, Li Q, Damjanovski S, Amano T, Ishizuya-Oka A: Regulation of apoptosis during development: input from the extracellular matrix (review). Int J Mol Med 1998, 2:273-282.

6. Elli L, Bergamini CM, Bardella MT, Schuppan D: Transglutaminases in inflammation and fibrosis of the gastrointestinal tract and the liver. Dig Liver Dis 2009, 41:541-550.

7. Darby I, Skalli O, Gabbiani G: Alpha-smooth muscle actin is transiently expressed by myofibroblasts during experimental wound healing. Lab Invest 1990, 63:21-29.

8. Sappino AP, Masouye I, Saurat JH, Gabbiani G: Smooth muscle differentiation in scleroderma fibroblastic cells. Am J Pathol 1990, 137:585-591.

9. Smith TJ: Insights into the role of fibroblasts in human autoimmune diseases. Clin Exp Immunol 2005, 141:388-397.

10. Villaschi S, Nicosia RF: Paracrine interactions between fibroblasts and endothelial cells in a serum-free coculture model. Modulation of angiogenesis and collagen gel contraction. Lab Invest 1994, 71:291-299.

11. Pang G, Couch L, Batey R, Clancy R, Cripps A: GM-CSF, IL-1 alpha, IL-1 beta, IL-6, IL-8, IL-10, ICAM-1 and VCAM-1 gene expression and cytokine production in human duodenal fibroblasts stimulated with lipopolysaccharide, IL-1 alpha and TNF-alpha. Clin Exp Immunol 1994, 96:437-443.

12. Saada JI, Pinchuk IV, Barrera CA, Adegboyega PA, Suarez G, Mifflin RC, Di Mari JF, Reyes VE, Powell DW: Subepithelial myofibroblasts are novel nonprofessional APCs in the human colonic mucosa. J Immunol 2006, 177:5968-5979.

13. Hogaboam CM, Smith RE, Kunkel SL: Dynamic interactions between lung fibroblasts and leukocytes: implications for fibrotic lung disease. Proc Assoc Am Physicians 1998, 110:313-320.

14. Khoo TK, Bahn RS: Pathogenesis of Graves' ophthalmopathy: the role of autoantibodies. Thyroid 2007, 17:1013-1018.

15. Elli L, Bardella MT: Motility disorders in patients with celiac disease. Scand J Gastroenterol 2005, 40:743-749.

16. Verbeke S, Gotteland M, Fernandez M, Bremer J, Rios G, Brunser O: Basement membrane and connective tissue proteins in intestinal mucosa of patients with coeliac disease. J Clin Pathol 2002, 55:440-445

17. Maiuri L, Ciacci C, Ricciardelli I, Vacca L, Raia V, Rispo A, Griffin M, Issekutz T, Quaratino S, Londei M: Unexpected role of surface transglutaminase type II in celiac disease. Gastroenterology 2005, 129:1400-1413.

18. Elli L, Dolfini E, Bardella MT: Gliadin cytotoxicity and in vitro cell cultures. Toxicol Lett 2003, 146:1-8.

19. Roncoroni L, Elli L, Doneda L, Piodi L, Ciulla MM, Paliotti R, Bardella MT: Isolation and culture of fibroblasts from endoscopic duodenal biopsies of celiac patients. J Transl Med 2009, 7:40.

20. Elli L, Roncoroni L, Doneda L, Ciulla MM, Colombo R, Braidotti P, Bonura A, Bardella MT: Imaging analysis of the gliadin direct effect on tight junctions in an in vitro three-dimensional Lovo cell line culture system. Toxicol In Vitro 2011, 25:45-50.

21. Koning F, Schuppan D, Cerf-Bensussan N, Sollid LM: Pathomechanisms in celiac disease. Best Pract Res Clin Gastroenterol 2005, 19:373-387.

22. Schuppan D: Current concepts of celiac disease pathogenesis. Gastroenterology 2000, 119:234-242.

23. Dieterich W, Esslinger B, Trapp D, Hahn E, Huff T, Seilmeier W, Wieser H, Schuppan D: Cross linking to tissue transglutaminase and collagen favours gliadin toxicity in coeliac disease. Gut 2006, 55:478-484. 
24. Ciccocioppo R, Di Sabatino A, Bauer M, Della Riccia DN, Bizzini F, Biagi F, Cifone MG, Corazza GR, Schuppan D: Matrix metalloproteinase pattern in celiac duodenal mucosa. Lab Invest 2005, 85:397-407.

25. Daum S, Bauer U, Foss HD, Schuppan D, Stein H, Riecken EO, Ullrich R: Increased expression of mRNA for matrix metalloproteinases-1 and -3 and tissue inhibitor of metalloproteinases- 1 in intestinal biopsy specimens from patients with coeliac disease. Gut 1999, 44:17-25.

26. Daum S, Bauer U, Foss HD, Wahnschaffe U, Schuppan D, Stein H, Riecken EO, Ullrich R: Expression of matrix metalloprotease-1 and collagen I mRNA in biopsies from patients with celiac disease. Ann N Y Acad Sci 1998, 859:254-257.

27. Motoyama T, Hojo $\mathrm{H}$, Watanabe $\mathrm{H}$ : Comparison of seven cell lines derived from human gastric carcinomas. Acta Pathol Jpn 1986, 36:65-83.

28. Sciaky D, Brazer W, Center DM, Cruikshank WW, Smith TJ: Cultured human fibroblasts express constitutive IL-16 mRNA: cytokine induction of active IL-16 protein synthesis through a caspase-3-dependent mechanism. J Immunol 2000, 164:3806-3814.

29. Korhonen M, Ormio M, Burgeson RE, Virtanen I, Savilahti E: Unaltered distribution of laminins, fibronectin, and tenascin in celiac intestinal mucosa. J Histochem Cytochem 2000, 48:1011-1020.

30. Mifflin RC, Pinchuk IV, Saada Jl, Powell DW: Intestinal myofibroblasts: targets for stem cell therapy. Am J Physiol Gastrointest Liver Physiol 2011, 300:G684-G696.

31. Barrera CA, Pinchuk IV, Saada Jl, Suarez G, Bland DA, Beswick E, Adegboyega PA, Mifflin RC, Powell DW, Reyes VE: Class II MHC-expressing myofibroblasts play a role in the immunopathogenesis associated with staphylococcal enterotoxins. Ann N Y Acad Sci 2004, 1029:313-318.

32. Mogilner A, Keren K: The shape of motile cells. Curr Biol 2009, 19:R762-R771.

33. Buonomo R, Giacco F, Vasaturo A, Caserta S, Guido S, Pagliara V, Garbi C, Mansueto G, Cassese A, Perruolo G, et al: PED/PEA-15 controls fibroblast motility and wound closure by ERK1/2-dependent mechanisms. J Cell Physiol 2012, 227:2106-2116.

34. Lerman OZ, Galiano RD, Armour M, Levine JP, Gurtner GC: Cellular dysfunction in the diabetic fibroblast: impairment in migration, vascular endothelial growth factor production, and response to hypoxia. Am J Pathol 2003, 162:303-312.

35. Busch R, De Riva A, Hadjinicolaou AV, Jiang W, Hou T, Mellins ED: On the perils of poor editing: regulation of peptide loading by HLA-DQ and H2A molecules associated with celiac disease and type 1 diabetes. Expert Rev Mol Med 2012, 14:e15.

doi:10.1186/1479-5876-11-9

Cite this article as: Roncoroni et al:: Extracellular matrix proteins and displacement of cultured fibroblasts from duodenal biopsies in celiac patients and controls. Journal of Translational Medicine 2013 11:91.

\section{Submit your next manuscript to BioMed Central and take full advantage of:}

- Convenient online submission

- Thorough peer review

- No space constraints or color figure charges

- Immediate publication on acceptance

- Inclusion in PubMed, CAS, Scopus and Google Scholar

- Research which is freely available for redistribution 